\title{
Towards Robust Deep Hiding Under Non-Differentiable Distortions for Practical Blind Watermarking
}

\author{
Chaoning Zhang*, Adil Karjauv*, Philipp Benz*, In So Kweon \\ Korea Advanced Institute of Science and Technology (KAIST) \\ Robotics and Computer Vision Lab, Daejeon, Korea \\ \{chaoningzhang1990,mikolez\}@gmail.com,\{pbenz,iskweon77\}@kaist.ac.kr
}

\begin{abstract}
Data hiding is one widely used approach for proving ownership through blind watermarking. Deep learning has been widely used in data hiding, for which inserting an attack simulation layer (ASL) after the watermarked image has been widely recognized as the most effective approach for improving the pipeline robustness against distortions. Despite its wide usage, the gain of enhanced robustness from ASL is usually interpreted through the lens of augmentation, while our work explores this gain from a new perspective by disentangling the forward and backward propagation of such ASL. We find that the main influential component is forward propagation instead of backward propagation. This observation motivates us to use forward ASL to make the pipeline compatible with nondifferentiable and/or black-box distortion, such as lossy (JPEG) compression and photoshop effects. Extensive experiments demonstrate the efficacy of our simple approach.
\end{abstract}

\section{CCS CONCEPTS}

- Security and privacy $\rightarrow$ Digital rights management;

\section{KEYWORDS}

Robust Deep Hiding; Lossy Compression; Non-differentiable; Blind Watermarking

\section{ACM Reference Format:}

Chaoning Zhang*, Adil Karjauv*, Philipp Benz*, In So Kweon. 2021. Towards Robust Deep Hiding Under Non-Differentiable Distortions for Practical Blind Watermarking. In Proceedings of the 29th ACM International Conference on Multimedia (MM '21), October 20-24, 2021, Virtual Event, China. ACM, New York, NY, USA, 9 pages. https://doi.org/10.1145/3474085.3475628

\section{INTRODUCTION}

The internet has become one of the most convenient and costeffective remote communication media [4]. While early internet users mainly communicated in plain text, in recent years, images or even videos have gradually become the dominant communication

\footnotetext{
${ }^{*}$ Equal contribution

Permission to make digital or hard copies of all or part of this work for personal or classroom use is granted without fee provided that copies are not made or distributed for profit or commercial advantage and that copies bear this notice and the full citation on the first page. Copyrights for components of this work owned by others than ACM must be honored. Abstracting with credit is permitted. To copy otherwise, or republish, to post on servers or to redistribute to lists, requires prior specific permission and/or a fee. Request permissions from permissions@acm.org.

MM '21, October 20-24, 2021, Virtual Event, China

(c) 2021 Association for Computing Machinery.

ACM ISBN 978-1-4503-8651-7/21/10 .\$15.00

https://doi.org/10.1145/3474085.3475628
}

media. However, those images and videos are vulnerable to unauthorized use. Data hiding has been established as a widely adopted approach for authentication and ownership protection [2]. Vast amounts of multimedia content in the form of images and video are shared daily on social media, such as Facebook, Twitter, and YouTube. To reduce traffic bandwidth, many photos and videos are compressed, e.g. through JPEG or MPEG. This kind of lossy compression can easily destroy the hidden watermark [4]. Thus, compression-resilient data hiding in multimedia has become a hot research direction $[2,16]$.

Recently, deep learning has also shown great success in hiding data in images [11,31,32], such as for the purpose of watermarking $[1,37]$. For improving robustness against distortions, the widely recognized simple yet effective approach is to insert an attack simulation layer (ASL) after the watermarked image during the training $[1,37]$. Despite its wide usage, the reason why ASL is effective is still rarely investigated. The gain of enhanced robustness is often interpreted through the lens of augmentation, while our work explores this robustness gain from a new perspective by disentangling the ASL in the forward and backward propagation. In essence, our work seeks to answer a question: does the forward or backward propagation of the ASL mainly contribute to the robustness gain? Through disentangling, we define forward ASL and backward ASL and find forward ASL achieves comparable (slightly inferior) performance as the standard ASL while backward ASL only increases the robustness marginally. Our results suggest that the main influential component of ASL is forward propagation instead of backward propagation. The superiority of standard ASL to forward ASL is empirically attributed to over-fitting.

The challenge of applying standard ASL to the deep hiding pipeline for improving the robustness against lossy compression is that they are non-differentiable. Inspired by the observation that forward ASL achieves comparable performance, we propose forward ASL as an alternative ASL approach to address this nondifferentiable challenge. We further demonstrate that forward ASL can be applied to black-box distortion, such as various photoshop effects. The proposed forward ASL can also be applied for being robust to various lossy compressions and/or photoshop effects. We further demonstrate that forward ASL can be readily extended to the task of hiding an image in another image [3] or hiding video in another video [22]. We are not the first one to attempt to address this non-differentiable challenge [1, 20, 37], however, our approach is simple, generalizable and also achieves superior performance. 


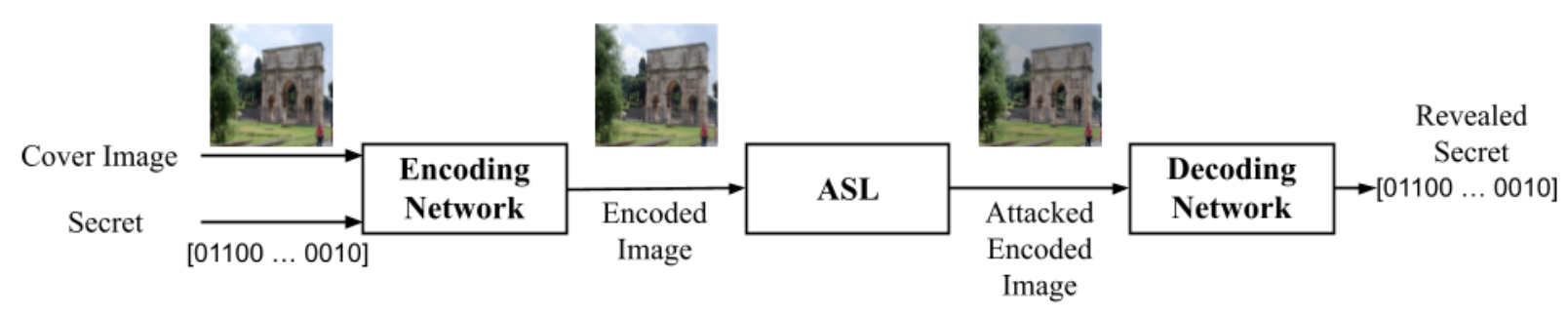

Figure 1: Main framework for robustly encoding and decoding secret messages.

\section{RELATED WORK}

\subsection{Deep Learning Based Robust Data Hiding}

For the general deep learning based data hiding, refer to [36] and here we summarize those specifically related to robust data hiding $[5,6]$. Early works of traditional methods have mainly explored manipulating the cover image pixels directly, i.e. embedding the hidden data in the spatial domain, for which hiding data in the least significant bits (LSBs) can be seen as a classical example. For improving robustness, the trend has shifted to hiding in a transform domain, such as Discrete Cosine Transform (DCT) [28], Discrete Wavelet Transform [21], or a mixture of them [10, 29]. Beyond the success in a wide range of applications, deep learning has found its application [13] in data hiding, demonstrating comparable or superior performance than traditional approaches in terms of capacity, robustness, and security [37]. The trend has shifted from adopting DNNs for a certain stage of the whole data hiding pipeline $[14,15,24]$ to utilizing DNNs for the whole pipeline in an end-to-end manner [11]. [3] has shown the possibility of hiding a full image in an image, which has been extended to hiding video in video in $[31,32]$. Hiding binary data with adversarial learning has also been proposed in [11]. The above deep learning approaches do not take robustness into account and the hidden data can easily get destroyed by common lossy compression, such as JPEG. Inserting an ASL module after the watermarked image during the training is widely recognized as the most effective approach to improve the robustness to the anticipated distortions. This approach works for most common distortions but not for nondifferentiable JPEG. To overcome the challenge that "true" JPEG is non-differentiable, the authors [37] propose to "differentiate" the JPEG compression with two approximation methods, namely JPEGMask and JPEG-Drop. There exists a substantial gap between them and "true" JPEG, resulting in poor performance when tested under the "true" JPEG. To minimize the gap approximation and "true" JPEG, more recently, [1] proposed a new approach to carefully simulate the important steps in the "true" JPEG. Two-stage separable training has been introduced in [20] to extend this beyond JPEG to general non-differentiable and/or black-box distortions.

\subsection{Lossy Data Compression Beyond JPEG}

Even though most works mainly analyze the influence of JPEG, there also exist other variants of lossy compression [26]. Since lossy compression is the primary distortion our proposed ASL approach attempts to address, here we present a brief summarization of them. JPEG compression involves two major steps: DCT transform and quantization. For color images, color transformation is also often adopted as a standard pre-processing step [25]. JPEG2000 [30] is another common lossy compression that has higher visual quality with fewer mosaic artifacts. In contrast to JPEG, JPEG2000 is based on Discrete Wavelet Transform [21]. In 2010, Google developed a new image format called WebP, which is similar to JPEG based on the DCT transform but outperforms JPEG in terms of compression ratio [9]. For video lossy compression, MPEG [18] is the most widely adopted approach.

\section{PRELIMINARY KNOWLEDGE}

The main objective is to design an end-to-end trainable architecture for a deep watermarking pipeline which exhibits reasonable robustness to various types of distortions. Our main framework closely follows the architecture presented in [20] and is shown in Figure 1. It comprises of four major components: (1) an encoding network $E$, parameterized through $\theta_{E}$, which receives a cover image $I_{C} \in \mathbb{R}^{H \times W \times C}$, where $H$ and $W$ represent the image size and $C=3$ for a color image, and a secret message $S \in\{0,1\}^{K}$ of length $K$ as input; it outputs an encoded image $I_{E} \in \mathbb{R}^{H \times W \times C}$; (2) the attack simulation layer (ASL) receives the cover image $I_{C}$ and the encoded image $I_{E}$ and applies an attack, which can be a distortion, e.g. JPEG compression, resulting in the attacked encoded image $I_{E}^{\prime}$; (3) the decoder network $D$, parameterized through $\theta_{D}$, receives $I_{E}^{\prime}$ and retrieves the secret message $S^{\prime}$ from it; (4) a discriminator T, parameterized through $\theta_{T}$, receives either a cover image $I_{C}$ or an encoded image $I_{E}$, with the goal to distinguish between the two through an output probability $\mathbf{T}(I) \in[0,1]$. Note that in Figure 1, the discriminator is not shown for simplicity. In the following, we provide details about these individual building blocks, followed by a description of the individual loss parts to train the deep watermarking pipeline.

Encoder. The encoder fulfills the task to embed the secret message into the cover image. It first transforms the cover image $I_{C}$ into an intermediate representation through a stack of convolutions. The secret is then duplicated along the height and width dimensions of this intermediate representation resulting in the channel dimension being equal to the secret length $K$. This secret message block is concatenated with the intermediate representation of the cover image and fed into a subsequent convolutional block. The output of the encoder is denoted as $I_{E}$.

Attack Simulation Layer (ASL). The ASL $[1,37]$ fulfills the purpose of increasing the robustness of the deep hiding pipeline to distortions/attack, e.g. random noise or JPEG compression. Therefore, the ASL receives $I_{E}$ and applies the attack, resulting in an attacked encoded image $I_{E}^{\prime}$. In this work, we analyze the influence 
of the forward and backward propagation of the ASL. We dedicate Section 4 toward a detailed discussion of the ASL.

Decoder. The purpose of the decoder is to retrieve the secret $S^{\prime}$ from the attacked encoded image $I_{E}^{\prime}$. It is comprised of several convolutional layers, transforming $I_{E}^{\prime}$ into a representation of $K$ channels, from which a vector of length $K$ is obtained through global spatial average pooling. Finally, a linear layer is applied to obtain the recovered secret $S^{\prime}$.

Discriminator. To additionally enhance the visual quality of the container image, a discriminator is used, distinguishing between the cover image and the encoded image. The architecture of the discriminator is similar to that of the decoder.

Loss Functions. The loss function consists of several parts. The first objective is to maintain a visual similarity between $I_{C}$ and $I_{E}$. Therefore, one part of the loss is responsible to minimize the distance between these two images, which is realized through an $\ell_{2}$-based loss:

$$
\mathcal{L}_{E 1}=\frac{\left\|I_{C}-I_{E}\right\|_{2}^{2}}{H W C}
$$

Additionally, a loss incorporating the discriminator is used, indicating the capability of the discriminator to identify the encoded image,

$$
\mathcal{L}_{E 2}\left(I_{E}\right)=\log \left(1-T\left(I_{E}\right)\right)
$$

Finally, the objective is also to retrieve the secret as accurately as possible. Therefore, a simple $\ell 2$-loss can be deployed.

$$
\mathcal{L}_{S}=\left\|S-S^{\prime}\right\|_{2}^{2}
$$

Given two weighting factors $\lambda_{E 1}$ and $\lambda_{E 2}$, the total loss is shown as follows:

$$
\mathcal{L}_{\text {total }}=\mathcal{L}_{S}+\lambda_{E 1} \mathcal{L}_{E 1}+\lambda_{E 2} \mathcal{L}_{E 2}
$$

The discriminator itself is trained through the following classification loss:

$$
\mathcal{L}_{T}=\log \left(1-T\left(I_{C}\right)\right)+\log \left(T\left(I_{E}\right)\right) .
$$

Metrics. To evaluate the effectiveness of the Deep Hiding architectures, we mainly use two metrics: the bit error rate (BER) [1] and the peak signal-to-noise ratio (PSNR) [12]. The BER is a measure of the deep watermarking pipeline robustness and is represented as the ratio of the number of non-equal bits between the secret $S$ and the revealed secret $S^{\prime}$ to the secret length. Since the secret is comprised of binary information, a BER of $50 \%$ equals a random guess. The PSNR measures the image distortion between the cover image and the encoded image and can be interpreted as the secrecy of the encoded image. For the BER, lower values are better, while for the PSNR, higher values are better. Naturally, a trade-off arises between the two metrics. To decrease the BER, often the secrecy has to be sacrificed and vice versa.

\section{DISENTANGLING ASL}

Despite the wide usage of the ASL [1,37], the reason behind the gain of enhanced robustness is rarely investigated. The basic motivation to insert such an ASL module can be somewhat interpreted through the lens of augmentation. For example, it is a common practice to improve the model generalization capability through augmenting samples with relevant augmentations, such as Gaussian noise. Commonly, an ASL is simply inserted into the pipeline and therefore incorporated into both forward and backward propagation. Here, we explore this robustness gain from a new perspective by disentangling the ASL into a forward and backward propagation. Note that [8] has analyzed the disentangling effect of dropout.

\subsection{Forward and Backward ASL}

We disentangle the ASL by proposing a forward ASL and a backward ASL. We will first provide a formal definition of the two and introduce their technical realization.

Forward ASL Definition. The encoded image is passed through the ASL before feeding it into the decoder but the gradient from the decoder is directly backpropagated to the encoder without passing through the ASL module.

Commonly, by simply plugging in an ASL module, the ASL would be automatically incorporated into the forward and backward propagation. As the name suggests, the forward ASL has the objective to influence the information propagation only in the forward propagation. Figure 2 (left) depicts the technical realization of this process. Given the encoded image, the resulting difference between the initial encoded image and the attacked encoded image can be obtained through subtraction of the encoded image from the attacked encoded image $\left(I_{E}^{\prime}-I_{E}\right)$. We indicate this difference as the pseudo-noise. By adding this pseudo-noise to the encoded image, the attacked encoded image is obtained, which would then be fed into the decoder network. During the backward propagation, the gradient flow is blocked for the lower part, indicated by the two bars. With this, the attacked encoded image can be obtained in the forward propagation, without the need to backpropagate gradients through the ASL module.

Backward ASL Definition. The encoded image is directly fed to the decoder but the gradient information from the decoder flows through the ASL before being back-propagated to the encoder.

Contrary to the forward ASL, the backward ASL is designed to only influence the information in the backward propagation. We visualize the technical realization of this scenario in Figure 2 (middle). During the forward propagation, the encoded image is fed into the ASL, resulting in the attacked encoded image. The pseudo-noise is calculated as before. By subtracting the pseudo-noise from the attacked encoded image the originally encoded image can again be obtained, which would then be subsequently fed into the decoder network. As for the forward-only propagation, the gradient is blocked from flowing through the lower part of the diagram, where the pseudo-noise is calculated. The gradient, however, flows through the ASL and would be further backpropagated to the encoder from there.

In summary, with the above descriptions, three variations of the ASL can be distinguished: forward ASL, backward ASL, and standard ASL. Through the disentanglement, we can investigate the effect of the forward or backward ASL on the robustness gain. To perform this analysis, the used ASL module needs to be differentiable; otherwise, the backward ASL cannot be realized. For simplicity and without losing generality, we choose random resize as the ASL module. The image can be resized either up to a larger resolution or down to a smaller resolution. We let the ASL include two sequential resize operations that first resize the image down to a smaller resolution with a scale factor (SF) and then resize it back to the original resolution. Specifically, we set the SF to 0.5 to train 


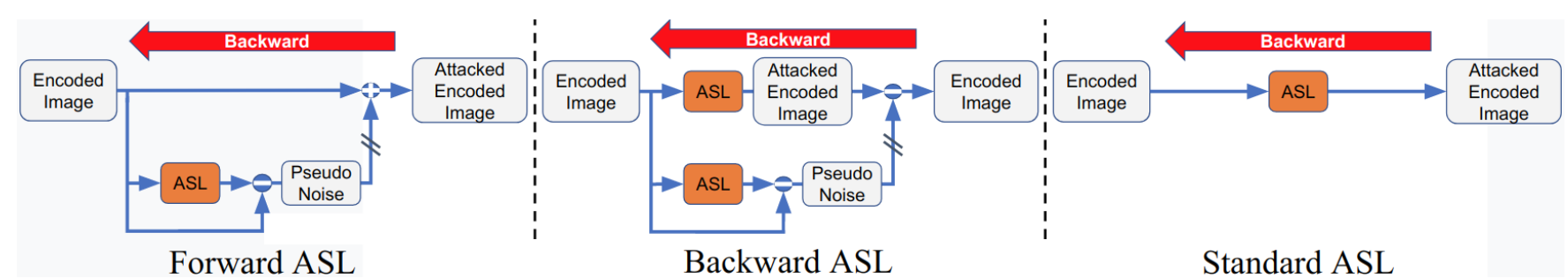

Figure 2: Three types of the attack simulation layer (ASL). $\backslash \backslash$ indicates a path that is only used in the forward propagation.

Table 1: Evaluation results of deep hiding pipeline trained with different ASLs: none, backward, forward, and standard. None indicates that no ASL is applied in the forward or backward propagation. The metric reported here is BER (\%). All models are trained with resize scale factor (SF) of 0.5 but evaluated on resize attack with a wide range of SFs.

\begin{tabular}{c|cccc}
\hline & None & Backward & Forward & Standard \\
\hline w/o resize & 0.01 & 3.01 & 7.08 & 10.78 \\
w/ resize (SF=0.2) & 49.11 & 47.90 & 43.67 & 44.07 \\
w/ resize (SF=0.3) & 45.93 & 47.80 & 38.54 & 38.56 \\
w/ resize (SF=0.4) & 36.60 & 37.20 & 16.66 & 19.30 \\
w/ resize (SF=0.5) & 30.17 & 29.11 & 3.99 & 3.22 \\
w/ resize (SF=0.6) & 21.01 & 23.05 & 6.80 & 8.36 \\
w/ resize $(\mathrm{SF}=0.7)$ & 16.05 & 12.39 & 5.50 & 8.07 \\
w/ resize $(\mathrm{SF}=0.8)$ & 11.98 & 8.09 & 4.92 & 5.61 \\
\hline
\end{tabular}

the deep hiding pipeline and evaluate the pipeline robustness under different SFs. The results are shown in Table 1. We find that under the resize attack, as expected, standard ASL yields significantly better performance than its counterpart without ASL under the resize attack with different SFs. The adopted resize attack with the SF of 0.5 is a strong distortion that leads to a BER of $30.17 \%$ when no ASL is applied during the training. Under such a strong resize attack, the BER with standard ASL is as low as $3.32 \%$, demonstrating the effectiveness of the widely used standard ASL approach. Further observation shows that backward-ASL leads to a marginal robustness boost with a BER of $29.11 \%$ (very close to $30.17 \%$ ) while forward-ASL yields a BER of $3.99 \%$ which is close to $3.22 \%$. The results suggest that the major factor that contributes to the robustness gain lies in the forward propagation, not the backward propagation.

Standard ASL vs. Forward ASL. We observe that backward ASL seems to not influence the pipeline performance when it alone is applied. However, when combined with the forward ASL, it still shows a visible performance boost, i.e. the BER of $3.22 \%$ vs. $3.99 \%$. Here, we seek to find the possible reason for the performance gap between standard ASL and forward ASL. In essence, inserting a standard ASL after the encoded image during the training is equivalent to adding a module in the pipeline, thus it is expected to be over-fitting to this specific module. On the other hand, forwardASL is equivalent to adding a pseudo-noise during the training. Thus we conjecture that it might be less over-fitting to this specific module compared with standard ASL. To verify this, we compare the performance of standard ASL and forward ASL under different SFs. We find that forward ASL achieves superior performance than standard ASL when the SF during the evaluation is different from
0.5. The results support our conjecture. Overall, we conclude (a) when the attack distortion is known and fixed, forward ASL can be slightly inferior to the standard ASL but forward ASL is still a competitive approach; (b) when the attack distortion during the inference changes, forward ASL is more appropriate.

\subsection{Forward ASL for Non-Differentiable Distortions}

One of the major drawbacks of the standard ASL is that it is only compatible with differentiable distortions. In practice, there are many types of non-differentiable (or even black-box) distortions and forward ASL without the need to apply ASL in the backward propagation is compatible with them. Inspired by the competitive performance of forward ASL compared with the standard ASL, we propose to adopt forward ASL as an alternative solution to improve the robustness of the deep watermarking pipeline, especially for the non-differentiable and/or black-box distortions. The PyTorch-like pseudo-code for forward ASL is shown in Algorithm 1. Note that this pseudo-code constitute as a Plug-and-Play module which can be easily inserted to existing deep watermarking pipeline.

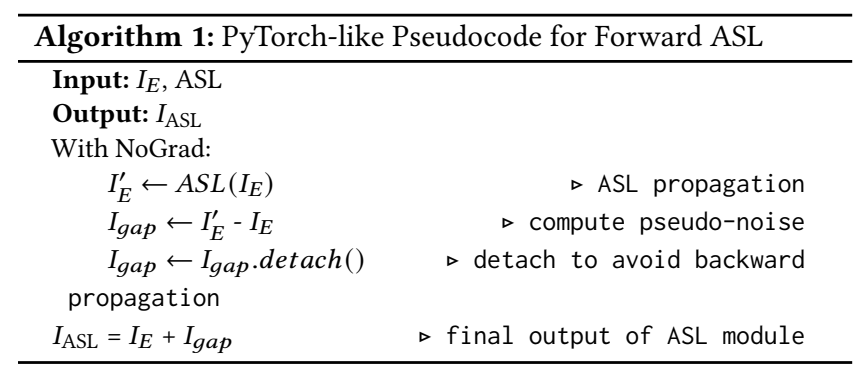

\section{MAIN RESULTS}

Our primary interest is to apply forward ASL to make the deep watermarking pipeline robust against lossy compression. Additionally, we also investigate its compatibility with black-box photoshop effects. We train each specified model to be robust against a single type of distortion; meanwhile, following [37] we apply different distortions in a combined manner to make the (combined) model robust against various distortions simultaneously. We use 10000 random images from the COCO dataset [19] for training the hiding pipeline and use separate images from COCO or ImageNet [7] for testing. Following [37], we hide 30 bits of random binary data in $128 \times 128$ color images. The weight factor $\lambda_{E 1}$ and $\lambda_{E 1}$ are set to 0.7 
and 0.001, respectively. We adopt the Adam [17] optimizer with a learning rate of $1 e-4$. We train the model for 200 epochs with a batch size of 32. Empirically, we find that for the distortion of photoshop effect, adopting the two-stage training as [20] stabilizes training. In contrast to [20] which keeps the encoder unchanged in the second stage, we further train the encoder but with a smaller learning rate of $1 e-6$. Note that we still keep the decoder learning rate $1 e-4$ unchanged in the second stage. For other types of distortions, we directly train them in a single-stage manner by directly applying forward ASL.

Lossy Compression. One of the primary focuses of this work is to make the deep watermarking pipeline robust against common corruptions. Among the common distortions, the lossy compression like JPEG is probably the most widely used one. Note that lossy compression by default is always non-differentiable due to many factors, such as quantization that involves rounding operations. An attacker can adopt various techniques, such as noising, filtering, or compression, to remove the hidden data. In practice, however, unauthorized sharing or modification is often conducted without an intentional attack. Instead, online platforms often perform an unintentional attack through lossy compression, while it is very unusual that a media platform would intentionally add noise to the images. Thus, addressing the robustness against lossy compression has high practical relevance. To our knowledge, prior works only investigate JPEG (see the comparison in Sec. 6.2), while our work also explores the relatively more recent JPEG-2000 and WebP compression algorithms. With the quality factor set to 90 , the results are shown in Table 2. Note, that even though the human eye can hardly notice the difference between the original image and the compressed one, lossy compression constitutes a very strong distortion attack. For example, when the pipeline is trained without the corresponding forward ASL, the investigated lossy compression deteriorates the robustness from a BER of $0 \%$ to higher than $40 \%$, close to $50 \%$ (random guess). Our forward ASL approach is effective to significantly improve the pipeline robustness. Under the forward ASL with specified lossy compression, the BERs for JPEG, JPEG2000 and WebP are $9.27 \%, 1.58 \%$, and $2.52 \%$, respectively. To make the pipeline robust against JPEG, JPEG2000, and WebP simultaneously, we combine all of the compression algorithms in the training and the resulting combined model shows reasonable robustness against all of them. Qualitative results for the three lossy compressions can be found in Figure 3.

Table 2: Robustness of the deep hiding pipeline against lossy compression. The averaged PSNR (dB) and BER (\%) are reported.

\begin{tabular}{c|c|cccc}
\hline & PSNR & Identity & JPEG & JPEG 2000 & WebP \\
\hline Identity & 44.07 & 0.00 & 47.63 & 43.50 & 44.09 \\
Forward JPEG & 27.84 & 20.51 & 9.27 & 35.23 & 9.10 \\
Forward JPEG 2000 & 28.03 & 4.98 & 25.32 & 1.58 & 21.83 \\
Forward WebP & 27.82 & 26.66 & 16.76 & 42.41 & 2.52 \\
Forward Combined & 28.40 & 0.02 & 10.19 & 10.57 & 5.54 \\
\hline
\end{tabular}

Photoshop Effects. To demonstrate the applicability of our forward ASL approach to black-box distortions, we experiment with

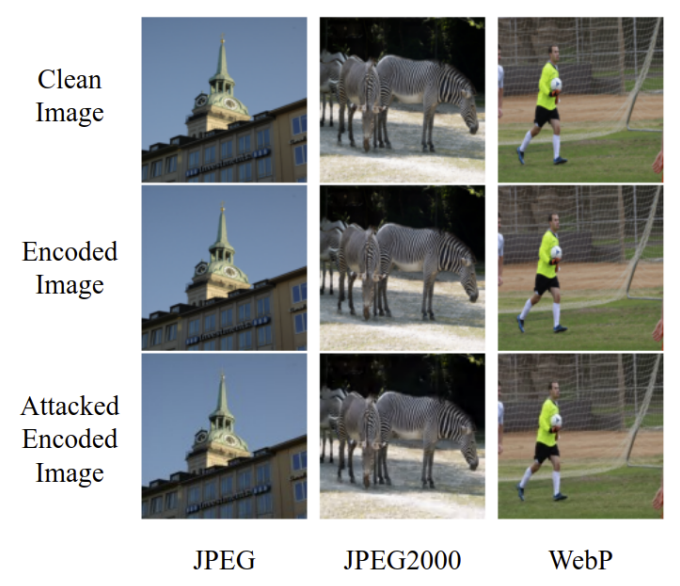

Figure 3: Qualitative results for the lossy compression distortions

various Photoshop effects. To not lose generality, we intentionally choose three photoshop effects with different properties: "country", which changes the color of the entire image in a similar manner; "solarize", which lets dark areas appear light and light areas appear dark; "wave", which changes the pixel position locally. Qualitative examples for the different photoshop effects applied to the encoded image are shown in Figure 4. The quantitative results are presented in Table 3. Applying the specified ASL during the training only improves the robustness against the corresponding distortion attack while the combined model is robust against all of them. It is worth mentioning that the photoshop effects behave as weaker distortion attack than lossy compression, even though the photoshop effects on the encoded image are more visible to the human eye.

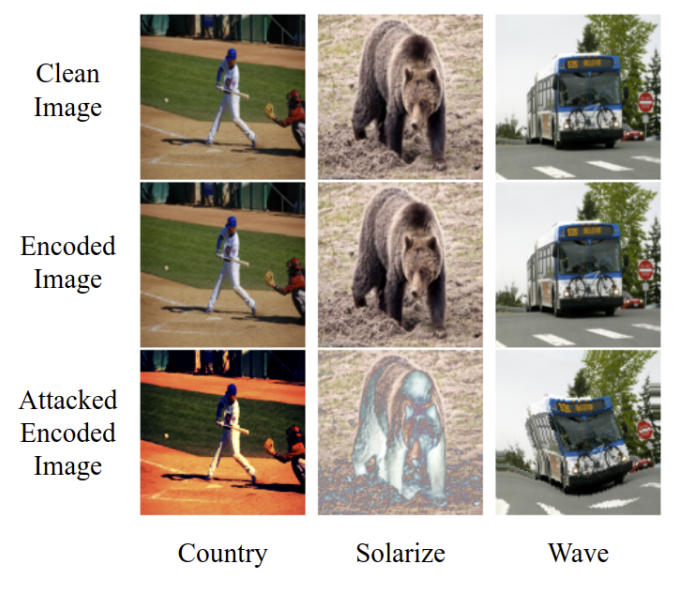

Figure 4: Qualitative results for the distortion of photoshop effects.

Joint Investigation of Photoshop Effects and Lossy Compression. We further train a combined model to make it robust against lossy compression and photoshop effects simultaneously. 
Table 3: Robustness of the deep hiding pipeline against photoshop effects. The averaged PSNR (dB) and BER (\%) are reported.

\begin{tabular}{c|c|cccc}
\hline & PSNR & Identity & Country & Solarize & Wave \\
\hline Identity & 44.07 & 0.00 & 6.79 & 28.50 & 17.20 \\
Forward Country & 37.63 & 0.02 & 0.09 & 30.38 & 17.41 \\
Forward Solarize & 34.56 & 8.52 & 26.49 & 2.41 & 29.17 \\
Forward Wave & 36.31 & 1.62 & 10.05 & 28.87 & 0.09 \\
Forward Combined & 35.80 & 0.09 & 0.52 & 2.56 & 1.51 \\
\hline
\end{tabular}

During training, one of the photoshop distortions or lossy compressions is randomly chosen and applied. However, during the inference stage, one photoshop effect and one lossy compression are applied sequentially to the encoded image. The results are shown in Table 4. We observe that the application of the forward ASL leads to a combined model with reasonable robustness against the joint distortion of photoshop effects and lossy compression. For example, with the joint distortion of country effect and JPEG compression, the model still has a BER of $24.42 \%$.

Table 4: Robustness of the deep hiding pipeline against joint distortion of photoshop effect (column) and lossy compression (row) in a sequential manner. The average BER (\%) is reported.

\begin{tabular}{c|cccc}
\hline & Identity & Country & Solarize & Wave \\
\hline Identity & 0.18 & 0.69 & 5.17 & 1.85 \\
JPEG & 12.64 & 24.42 & 33.91 & 32.87 \\
JPEG2000 & 13.47 & 22.29 & 27.51 & 25.08 \\
WebP & 8.10 & 20.39 & 30.07 & 26.28 \\
\hline
\end{tabular}

\section{ANALYSIS AND DISCUSSION}

\subsection{Extension to Other Domains}

Hiding an image in another image. [3] shows that deep learning can be applied to hide a full-color image in another. Here, we extend forward ASL to the context of an image being the secret instead of multiple secret bits. Specifically, we apply forward ASL to a pipeline that resembles [3] to make it simultaneously robust against JPEG, JPEG2000, and WebP. We measure the average pixel discrepancy (APD) between cover and container (encoded) images and that between secret and revealed secret images. The APD can simply be expressed as the $\ell 1$-norm. The APDs for them are 9.13/255 and $8.54 / 255$, respectively. The qualitative results are shown in Figure 5 and we observe that the content of the revealed secret image can still be observed. Note that naively training a pipeline without our forward ASL will result in a secret APD higher than 100/255, suggesting a failure of extracting the secret. Note that our technique has also previously shown to be compatible with universal deep hiding [35] for facilitating improving their robustness against JPEG.

Hiding Video in Video. Similar to hiding an image in an image, as shown above, we also experiment with hiding a video in video [22]. Given the container (encoded) video, we apply video compression to it. Specifically, we chose the commonly used MPEG4 (Part 14) as the compression algorithm. The qualitative results in

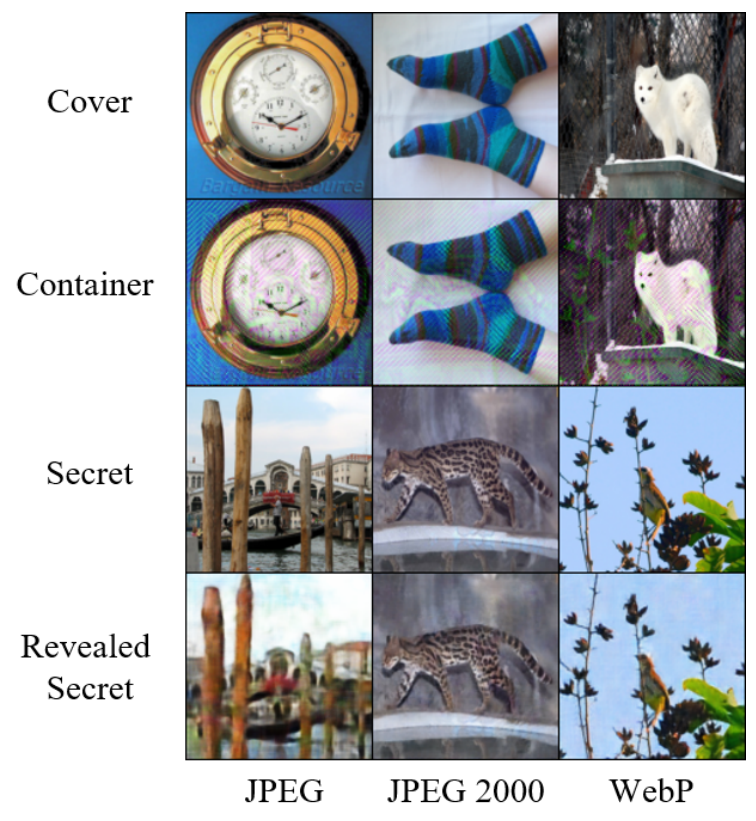

Figure 5: Hiding an image in another under different lossy compressions applied to the container (encoded) image.

Figure 6 show that without applying our forward ASL (MPEG), the decoder fails to extract any secret image content, demonstrating the necessity of our forward ASL. The results demonstrate our forward ASL approach is also compatible with video compression. To our knowledge, this is the first attempt in this direction for a deep watermarking pipeline.

JPEG Resistant Adversarial Examples. JPEG is one common defense method that alleviates the concern of adversarial attack [27]. [27] provides a solution to this through approximating JPEG with differentiable operations. Even though the primary focus of this work is to apply forward ASL for robust deep watermarking, forward ASL can also be used to generate JPEG-resistant adversarial examples. The results comparing our forward ASL with [27] are shown in Table 5. We observe that our approach outperforms [27] in most cases, especially when the JPEG quality factor is set high. Note that in practice, JPEG quality factor is rarely set to lower than 90; otherwise the model clean accuracy would have a serious drop.

\subsection{Comparison with the Existing Baselines}

We are not the first one to solve this challenge of non-differentiable distortion in robust data hiding. The existing attempts can be summarized into two categories: distortion approximation and twostage separable training. Since most of them have worked exclusively or mainly on JPEG distortion, we will summarize and compare the two approaches with the JPEG distortion as an example.

Distortion Approximation. Take JPEG for example, as a lossy compression it is non-differentiable. This renders JPEG not compatible with gradient-based optimization. In essence, JPEG limits the amount of information that can be transmitted, inspired by which [37] investigates approximating the JPEG operation by using 

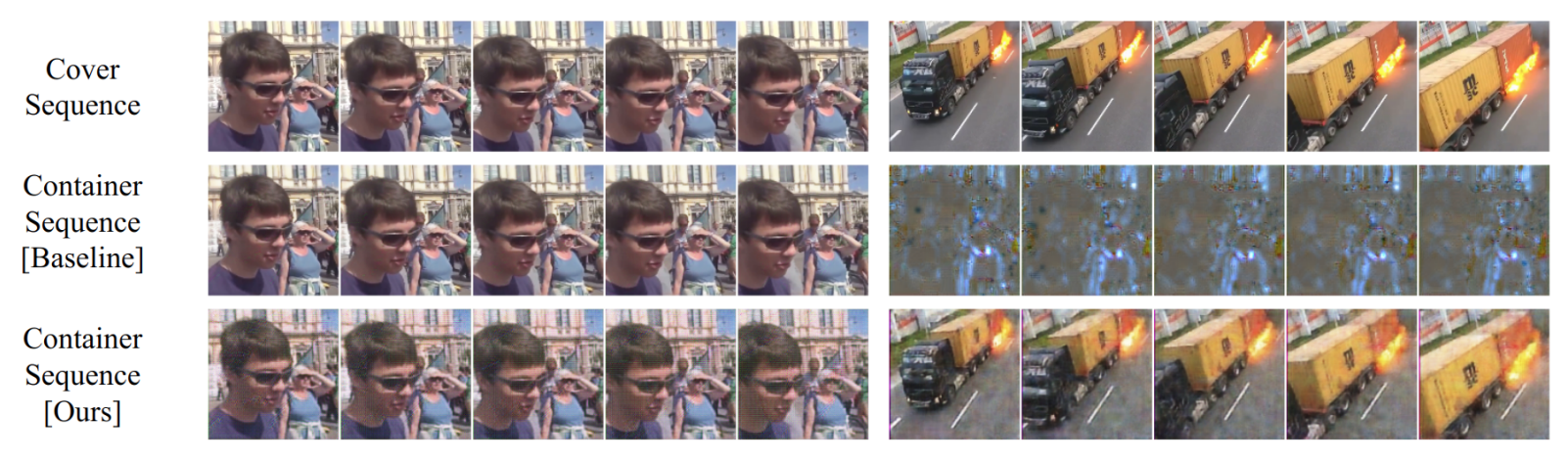

Secret

Sequence

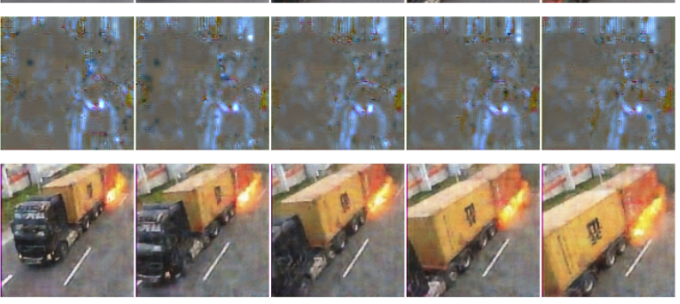

Revealed

Sequence

[Baseline]

Revealed

Sequence

[Ours]

Figure 6: Qualitative results for the task of hiding a video inside another video. In the first row, two examples of video sequences are shown: cover ( 5 frames to the left) and secret ( 5 frames to the right). The secret is then hidden inside the cover sequence via an encoder, which results in a container sequence. Container sequences for baseline encoder and encoder trained with our method are shown in the second and third rows to the left, respectively. The respective revealed secrets are shown in the second and the third rows to the right. The baseline decoder fails to reveal the hidden secret sequence of frames, while ours successfully retrieves the video frames.

Table 5: JPEG-resistant targeted adversarial examples. The rows indicate the quality factor $q$ for which the adversarial example was crafted, while the columns indicate the q's used for evaluation. The reported values are targeted attack success rate (higher is better). Each entry has two values: the left one indicates the performance of [27] and the right one indicates that of our forward ASL.

\begin{tabular}{c|ccccc}
\hline \multicolumn{5}{c}{} & \multicolumn{5}{c}{ Evaluation } \\
& $\mathrm{q}=50$ & $\mathrm{q}=60$ & $\mathrm{q}=70$ & $\mathrm{q}=80$ & $\mathrm{q}=90$ \\
\hline $\mathrm{q}=50$ & $\mathbf{8 7 . 0 0} / 73.10$ & $\mathbf{5 6 . 9 0} / 37.40$ & $\mathbf{3 3 . 9 0} / 24.80$ & $25.00 / \mathbf{2 6 . 7 0}$ & $11.00 / \mathbf{2 5 . 2 0}$ \\
$\mathrm{q}=60$ & $54.60 / \mathbf{5 7 . 8 0}$ & $\mathbf{8 7 . 8 0} / 84.80$ & $\mathbf{6 2 . 1 0} / 52.30$ & $\mathbf{4 6 . 0 0} / 42.30$ & $20.30 / \mathbf{4 1 . 7 0}$ \\
$\mathrm{q}=70$ & $7.80 / \mathbf{2 8 . 2 0}$ & $52.10 / \mathbf{6 9 . 4 0}$ & $92.60 / \mathbf{9 4 . 9 0}$ & $63.90 / \mathbf{7 3 . 0 0}$ & $39.40 / \mathbf{7 3 . 9 0}$ \\
$\mathrm{q}=80$ & $1.50 / \mathbf{9 . 6 0}$ & $2.70 / \mathbf{2 6 . 7 0}$ & $44.00 / \mathbf{7 9 . 9 0}$ & $95.90 / \mathbf{9 9 . 2 0}$ & $78.00 / \mathbf{9 1 . 6 0}$ \\
$\mathrm{q}=90$ & $0.80 / \mathbf{1 . 9 0}$ & $1.50 / \mathbf{4 . 9 0}$ & $6.50 / \mathbf{2 5 . 9 0}$ & $17.60 / \mathbf{7 9 . 4 0}$ & $97.70 / \mathbf{9 9 . 0 0}$ \\
\hline
\end{tabular}

a convolution layer with the stride of $8 \times 8$ as a substitute for the DCT transformation. Masking/Dropout is then applied to the resulting network activations that represent DCT domain coefficients. The two approximation approaches are coined as JPEG-Mask and JPEG-Drop. Specifically, JPEG-mask applies fixed masking that only keeps the low-frequency coefficients while JPEG-Drop randomly drops some coefficients but with a higher dropout rate for the high-efficiency coefficients in a progressive manner. [1] carefully designs every step in the JPEG and approximates them with the same functions except for the quantization part.

Two-Stage Separable Deep Learning (TSDL). The TSDL approach has been proposed by [20]. Specifically, it divides the training into two stages: namely noise-Free End-to-end Adversarial Training (FEAT) and noise-Aware Decoder-Only Training (ADOT). In the first FEAT stage, it trains the encoder and decoder without any distortion that leads to an encoder responsible for embedding the watermark. In the second stage, it fixes the weights of the pretrained-encoder but continues to train the decoder after applying the relevant non-differentiable ASL. Note that in the second stage, the gradient does not need to flow through the encoder so that there is no barrier in the training even the distortion is non-differentiable. Compared with the first baseline of distortion approximation, this two-stage training approach addresses this problem in an elegant manner that does not require engineering effort to approximate those steps in the distortions and it can be easily extended to any distortion even for black-box distortions.

Table 6: Comparison of our forward ASL method with SOTA baselines for improving the robustness against JPEG. ReDMark [1] can be seen as an improved version of HiDDeN [37] because [1] carefully approximates every step in JPEG with differentiable operations. The results are reported with the metric of BER (\%).

\begin{tabular}{cccccc}
\hline JPEG Quality & Baseline & HiDDeN [37] & ReDMark [1] & TSDL [20] & Ours \\
\hline $\mathrm{q}=10$ & 49.93 & 46.38 & 35.68 & 48.41 & $\mathbf{3 1 . 2 2}$ \\
$\mathrm{q}=25$ & 49.89 & 41.38 & 22.00 & 44.19 & $\mathbf{1 6 . 7 5}$ \\
$\mathrm{q}=50$ & 49.67 & 38.48 & 15.85 & 39.95 & $\mathbf{1 2 . 6 3}$ \\
$\mathrm{q}=75$ & 49.37 & 37.10 & 14.03 & 37.77 & $\mathbf{1 2 . 4 1}$ \\
$\mathrm{q}=90$ & 47.48 & 34.93 & 13.81 & 36.26 & $\mathbf{1 2 . 0 0}$ \\
\hline
\end{tabular}

Comparison. Requiring a non-trivial amount of engineering effort, the distortion approximation method is only applicable for white-box distortion that can be well approximated by differentiable functions. TSDL works for all types of distortions but has a distortion-unaware encoder. A comparison with existing JPEG approximation methods is shown in Table 6 where all the models are trained on JPEG-50 but evaluated on a wide range of quality factors. Our method outperforms all existing techniques for different JPEG quality factors. Note that distortion approximation based on ReDMark [1] also achieves competitive performance, but it cannot be readily adapted to non-differentiable distortions. Our proposed approach has the same applicability as TSDL but leads to a distortion-aware encoder, which can significantly improve the performance under serious distortions (see the comparison between TSDL [20] and ours in Table 6). Moreover, similar to the baseline of distortion approximation, our approach can be trained in an 
end-to-end single-stage manner. The core difference between TSDL and our approach lies in whether the encoder is distortion-aware. The merit of TSDL keeping the encoder distortion-unaware is that the encoder image quality would be higher. Note that the quality of the encoded image is solely determined by the encoder and thus does not change during the second stage when the encoder weights are fixed. Our approach making the encoder distortion-aware introduces more flexibility for the encoder to adapt to embed the secret message in a manner that is not easily destroyed by the distortion. Empirically we find that this distortion-aware mechanism often decreases the container image quality but achieves a significantly low BER, especially under strong distortion. In this sense, the comparison of our approach and TSDL [20] is not fair because their container image has higher quality. [20] introduced a strength factor $S$ to control the strength of the embedded watermark. Specifically, their encoder produces only the encoding of the watermark $I_{m}$, which is added to the cover image, hence their encoded image results in $I_{E}=I_{C}+S \cdot I_{m}$. We use different strength factors to make it have the same PSNR as ours and we indicate our result as a star in Figure 7. The result shows that this practice indeed decreases their BER but the gap between their BER and ours is still large. One additional concern is that their decoder is not trained with the increased strength factor, which might influence their performance. To this end, we further retrain their decoder with a respective strength factor of 5 in the second stage and indicate this result with a dot in Figure 7. This can further decrease their BER to $25.78 \%$, but is still higher than our $12.63 \%$. We also compare our approach with TSDL for a combination of distortion sets. As expected, the results in Table 7 show that ours outperform TSDL [20] by a large margin for most distortions except for Cropout and Crop.

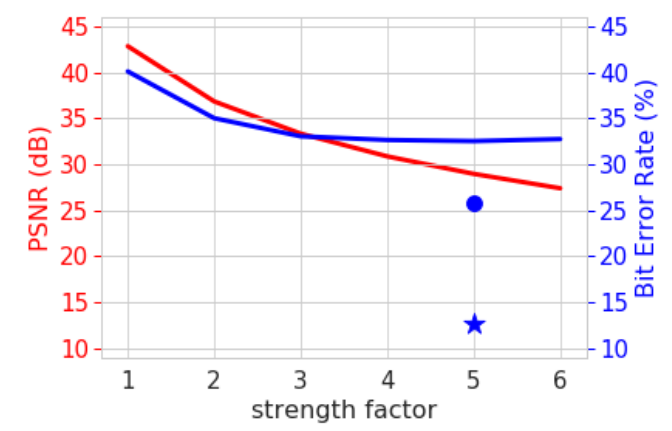

Figure 7: Evaluation of the TSDL approach for different strength factors for the PSNR (red) and BER (blue). Our BER is indicated as a star aligned with the PSNR value of TSDL, which exhibits the lowest BER value. The BER value of TSDL for a decoder trained with a strength factor of 5 is shown as a dot.

Additionally, we also compare our approach with a traditional method [16] which achieves a BER of $14.6 \%$ under the JPEG corruption (Quality factor 50) while hiding 30 bits in a $128 \times 128$ color images. Our approach achieves a comparable performance of $12.63 \%$ (see Table 6). As pointed out in [3,35], deep learning based approaches have the advantage of hiding significantly more information, such as hiding a full image or video in another image or video (see Sec. 6.1), while traditional methods typically only work well for hiding simple bits of secret message.

Table 7: Additional comparison with TSDL on a combination of distortions.

\begin{tabular}{ccccccccc}
\hline & Dropout & Cropout & Crop & Gaussian blur & JPEG & Resize & Gaussian noise & Salt\&Pepper \\
\hline TSDL [20] & 1.60 & 26.29 & 41.31 & 18.49 & 43.75 & 18.91 & 41.70 & 11.03 \\
Ours & 0.09 & 26.88 & 41.66 & 4.89 & 25.59 & 1.86 & 1.41 & 0.01 \\
\hline
\end{tabular}

\subsection{Frequency Perspective On An Intriguing Phenomenon}

One intriguing phenomenon observed from Sec. 5 is that photoshop effects overall cause more visual change to the container image but constitute a much weaker distortion attack than lossy compressions. We attempt to explain this phenomenon from the frequency perspective. The results in Figure 8 show that the secret is embedded in the form of high-frequency. Given that lossy compression, such as JPEG, is widely recognized to tend to remove high frequency, the occurrence of this phenomenon seems to be reasonable because photoshop effects mainly change the low-frequency content on the encoded image. This might also explain why increasing the robustness to one type of lossy compression also improves the robustness to another type of lossy compression (see Table 2). This frequency perspective is partly inspired by [34] for explaining UAP [23, 33] through the lens of deep steganography.

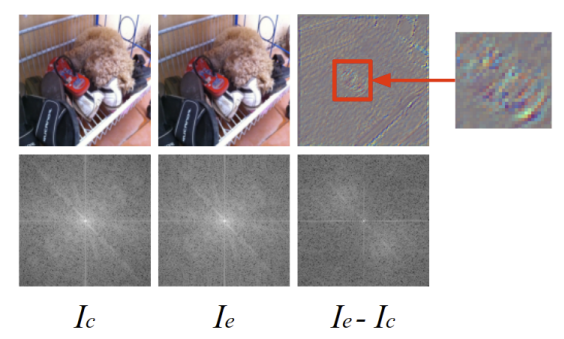

Figure 8: Frequency analysis. $I_{e}$ stands for an encoded image, $I_{c}$ indicates a clean image, and $I_{e}-I_{c}$ is a difference between encoded and clean images. A close-up view shows that the difference between these images is mainly in spatial highfrequencies.

\section{CONCLUSION}

Our work disentangles the widely used ASL in the forward and backward propagation, resulting in forward ASL and backward ASL. We find that forward ASL achieves comparable performance as standard ASL, inspired by which we propose to adopt forward ASL as a simple yet effective approach for improving the deep watermarking pipeline robust against non-differentiable and/or black-box distortions, such as lossy compression and photoshop effects. Extensive experiments demonstrate the efficacy of our approach with competitive performance against existing methods. 


\section{REFERENCES}

[1] Mahdi Ahmadi, Alireza Norouzi, Nader Karimi, Shadrokh Samavi, and Ali Emami 2020. ReDMark: Framework for residual diffusion watermarking based on deep networks. Expert Systems with Applications (2020).

[2] Farhan A Alenizi. 2017. Robust Data Hiding in Multimedia for Authentication and Ownership Protection. Ph.D. Dissertation.

[3] Shumeet Baluja. 2017. Hiding images in plain sight: Deep steganography. In NeurIPS.

[4] Z Bao, X Luo, Y Zhang, C Yang, and F Liu. 2018. A robust image steganography on resisting JPEG compression with no side information. IETE Technical Review (2018).

[5] Preeti Bhinder, Kulbir Singh, and Neeru Jindal. 2018. Image-adaptive watermarking using maximum likelihood decoder for medical images. Multimedia Tools and Applications (2018)

[6] Beijing Chen, Chunfei Zhou, Byeungwoo Jeon, Yuhui Zheng, and Jinwei Wang. 2018. Quaternion discrete fractional random transform for color image adaptive watermarking. Multimedia Tools and Applications (2018)

[7] Jia Deng, Wei Dong, Richard Socher, Li-Jia Li, Kai Li, and Li Fei-Fei. 2009. ImageNet: A large-scale hierarchical image database. In CVPR.

[8] Hongchang Gao, Jian Pei, and Heng Huang. 2019. Demystifying dropout. In ICML.

[9] Giaime Ginesu, Maurizio Pintus, and Daniele D Giusto. 2012. Objective assessment of the WebP image coding algorithm. Signal Processing: Image Communication (2012).

[10] NJ Harish, BBS Kumar, and Ashok Kusagur. 2013. Hybrid robust watermarking techniques based on DWT, DCT, and SVD. International fournal of Advanced Electrical and electronics engineering (2013).

[11] Jamie Hayes and George Danezis. 2017. Generating steganographic images via adversarial training. In NeurIPS.

[12] Quan Huynh-Thu and Mohammed Ghanbari. 2008. Scope of validity of PSNR in image/video quality assessment. Electronics letters (2008).

[13] Bibi Isac and V Santhi. 2011. A study on digital image and video watermarking schemes using neural networks. International Journal of Computer Applications (2011).

[14] Cong Jin and Shihui Wang. 2007. Applications of a neural network to estimate watermark embedding strength. In International Workshop on Image Analysis for Multimedia Interactive Services (WIAMIS).

[15] Haribabu Kandi, Deepak Mishra, and Subrahmanyam RK Sai Gorthi. 2017. Exploring the learning capabilities of convolutional neural networks for robust image watermarking. Computers \& Security (2017).

[16] Xiangui Kang, Jiwu Huang, and Wenjun Zeng. 2010. Efficient general printscanning resilient data hiding based on uniform log-polar mapping. TIFS (2010).

[17] Diederik P Kingma and Jimmy Ba. 2015. Adam: A method for stochastic optimization. In ICLR.

[18] Didier Le Gall. 1991. MPEG: A video compression standard for multimedia applications. Commun. ACM (1991).

[19] Tsung-Yi Lin, Michael Maire, Serge Belongie, James Hays, Pietro Perona, Deva Ramanan, Piotr Dollár, and C Lawrence Zitnick. 2014. Microsoft COCO: Common objects in context. In ECCV.

[20] Yang Liu, Mengxi Guo, Jian Zhang, Yuesheng Zhu, and Xiaodong Xie. 2019. A novel two-stage separable deep learning framework for practical blind watermarking. In $A C M M M$.

[21] Wei Lu, Wei Sun, and Hongtao Lu. 2009. Robust watermarking based on DWT and nonnegative matrix factorization. Computers \& Electrical Engineering (2009).

[22] Aayush Mishra, Suraj Kumar, Aditya Nigam, and Saiful Islam. 2019. VStegNET: Video Steganography Network using Spatio-Temporal features and MicroBottleneck. BMVC (2019).

[23] Seyed-Mohsen Moosavi-Dezfooli, Alhussein Fawzi, Omar Fawzi, and Pascal Frossard. 2017. Universal adversarial perturbations. In CVPR.

[24] Seung-Min Mun, Seung-Hun Nam, Han-Ul Jang, Dongkyu Kim, and Heung-Kyu Lee. 2017. A robust blind watermarking using convolutional neural network. arXiv preprint arXiv:1704.03248 (2017).

[25] William B Pennebaker and Joan L Mitchell. 1992. 7PEG: Still image data compression standard.

[26] Khalid Sayood. 2017. Introduction to data compression.

[27] Richard Shin and Dawn Song. 2017. Jpeg-resistant adversarial images. In NIPS 2017 Workshop on Machine Learning and Computer Security.

[28] Satendra Pal Singh and Gaurav Bhatnagar. 2018. A new robust watermarking system in integer DCT domain. Journal of Visual Communication and Image Representation (2018)

[29] Surya Pratap Singh, Paresh Rawat, and Sudhir Agrawal. 2012. A robust watermarking approach using DCT-DWT. International journal of emerging technology and advanced engineering (2012).

[30] Athanassios Skodras, Charilaos Christopoulos, and Touradj Ebrahimi. 2001. The jpeg 2000 still image compression standard. IEEE Signal processing magazine (2001)

[31] Xinyu Weng, Yongzhi Li, Lu Chi, and Yadong Mu. 2018. Convolutional video steganography with temporal residual modeling. arXiv preprint arXiv:1806.02941 (2018).

[32] Pin Wu, Yang Yang, and Xiaoqiang Li. 2018. Image-into-Image Steganography Using Deep Convolutional Network. In Pacific Rim Conference on Multimedia.

[33] Chaoning Zhang, Philipp Benz, Tooba Imtiaz, and In-So Kweon. 2020. Understanding Adversarial Examples from the Mutual Influence of Images and Perturbations. In CVPR.

[34] Chaoning Zhang, Philipp Benz, Adil Karjauv, and In So Kweon. 2021. Universal Adversarial Perturbations Through the Lens of Deep Steganography: Towards A Fourier Perspective. AAAI (2021).

[35] Chaoning Zhang, Philipp Benz, Adil Karjauv, Geng Sun, and In Kweon. 2020. UDH: Universal Deep Hiding for Steganography, Watermarking, and Light Field Messaging. NeurIPS (2020).

[36] Chaoning Zhang, Chenguo Lin, Philipp Benz, Kejiang Chen, Weiming Zhang, and In So Kweon. 2021. A Brief Survey on Deep Learning Based Data Hiding, Steganography and Watermarking. arXiv preprint arXiv:2103.01607 (2021).

[37] Jiren Zhu, Russell Kaplan, Justin Johnson, and Li Fei-Fei. 2018. Hidden: Hiding data with deep networks. In ECCV. 